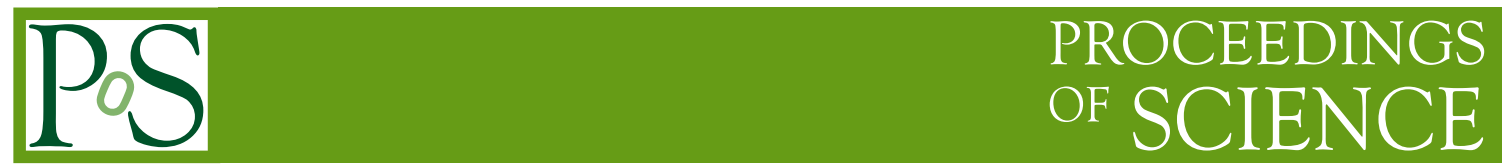

\title{
Lepton Flavor Violation and Dilepton Tails at the LHC
}

\section{Olcyr Sumensari ${ }^{a, *}$}

${ }^{a}$ Physik-Institut, Universität Zürich, CH-8057 Zürich, Switzerland

E-mail: olcyr.sumensari@physik.uzh.ch

Charged Lepton Flavor Violation (LFV) is a very clean probe of New Physics since it is forbidden in the Standard Model (SM). The observation of neutrino oscillation implies nonzero LFV rates, which however are highly suppressed by the smallness of neutrino masses. This makes LFV an appealing target of experimental searches, as its observation would unambiguously point to New Physics. In this proceedings, we will discuss the constraints on LFV effective operators that can be derived from LHC data on dilepton production. We will show that semileptonic operators can be constrained by existing searches of $p p \rightarrow \ell_{i} \ell_{j}$ (with $i \neq j$ ) at high- $p_{T}$. We will explore the complementarity of these constraints with the ones obtained from low-energy observables, by showing, in particular, that LHC data provides the best constraints for several quark-flavor conserving effective coefficients, as well as for the ones that are relevant for charm physics.

40th International Conference on High Energy physics - ICHEP2020

July 28 - August 6, 2020

Prague, Czech Republic (virtual meeting)

${ }^{*}$ Speaker 


\section{Introduction}

The Standard Model (SM) Lagrangian has an accidental symmetry $U(1)_{e} \times U(1)_{\mu} \times U(1)_{\tau}$, which guarantees the conservation of individual lepton flavor numbers and which must be broken since neutrinos are massive and oscillate. However, the smallness of neutrino masses ensures that all decays with Lepton Flavor Violation (LFV) are highly suppressed and thus not observable, in the absence of further contributions beyond the SM. For this reason, these processes are very clean probes of New Physics effects.

Several extensions of the SM predict potentially large effects in LFV processes, see e.g. Ref. [1] for a review. These effects can appear in purely leptonic decays, such as $\ell_{i}^{-} \rightarrow \ell_{j}^{-} \gamma$ and $\ell_{i}^{-} \rightarrow \ell_{j}^{-} \ell_{k}^{+} \ell_{k}^{-}$, with $i>j \geq k$, which are the target of several ongoing and future experiments in the lepton sector [2]. LFV in semileptonic transitions $q_{i} \rightarrow q_{j} \ell_{k}^{+} \ell_{l}^{-}$are also compelling probes of New Physics. These effects would be predominant, for instance, in scenarios with leptoquark states or new $Z^{\prime}$ bosons, as they could be induce these effects already at tree-level, see e.g. Ref. [3]. Presently, there is a rich experimental program dedicated to these processes at NA62 [4], BES-III [5], and LHCb [6] and Belle-II [7], which will greatly improve the experimental sensitivity on LFV decays of $K$-, $D$ - and $B$-mesons in the coming years.

LHC searches also offer new possibilities to constrain flavor physics at high- $p_{T}[8,9]$. The main idea here is to exploit the energy-enhancement of the dilepton-production cross-section in the presence of higher-dimension operators. Complementary constraints to low-energy probes can be derived in these studies, as explored e.g. in the context of the discrepancies observed in $B$-meson decays [10]. In this proceedings, we discuss the limits on semileptonic LFV operators that can be derived by using LHC bounds on the high- $p_{T}$ tails of the processes $p p \rightarrow \ell_{k} \ell_{l}$ with $k \neq l$ [11].

\section{EFT description}

First, we define our Effective Field Theory (EFT) and provide general expressions for $\sigma(p p \rightarrow$ $\ell_{k}^{ \pm} \ell_{l}^{\mp}$ ) within this framework. We consider the following effective Lagrangian,

$$
\mathcal{L}_{\text {eff }} \supset \sum_{\alpha} \sum_{i j k l} \frac{C_{\alpha}^{i j k l}}{v^{2}} O_{\alpha}^{i j k l},
$$

where $v=\left(\sqrt{2} G_{f}\right)^{-1 / 2}$ is the electroweak vacuum expectation value, $O_{\alpha}^{i j k l}$ are the semi-leptonic operators listed in Table 1 and $C_{\alpha}^{i j k l}$ are effective coefficients. In our notation, $q_{i, j}$ can denote either up or down-type quarks. The matching of Eq. (1) to the SM EFT is provided in Ref. [11]. By neglecting the fermion masses, we can generically express the differential partonic cross-section for this process as follows

$$
\begin{aligned}
{\left[\frac{\mathrm{d} \hat{\sigma}}{\mathrm{d} \hat{t}}\right]_{i j k l}=\frac{1}{48 \pi v^{4} \hat{s}^{2}} } & \left\{\hat{t}^{2}\left[\left|C_{V_{L R}}\right|^{2}+\left|C_{V_{R L}}\right|^{2}\right]+(\hat{s}+\hat{t})^{2}\left[\left|C_{V_{L L}}\right|^{2}+\left|C_{V_{R R}}\right|^{2}\right]\right. \\
& \left.+\frac{\hat{s}^{2}}{4}\left[\left|C_{S_{L}}\right|^{2}+\left|C_{S_{R}}\right|^{2}\right]+4(\hat{s}+2 \hat{t})^{2}\left|C_{T}\right|^{2}-2 \hat{s}(\hat{s}+2 \hat{t}) \operatorname{Re}\left(C_{S_{L}} C_{T}^{*}\right)\right\}
\end{aligned}
$$




\begin{tabular}{|c|c||c|}
\hline Eff. coeff. & Operator & SMEFT \\
\hline \hline$C_{V_{L L}}^{i j k l}$ & $\left(\bar{q}_{L i} \gamma_{\mu} q_{L j}\right)\left(\bar{\ell}_{L k} \gamma^{\mu} \ell_{L l}\right)$ & $O_{l q}^{(1)}, O_{l q}^{(3)}$ \\
$C_{V_{R R}}^{i j k l}$ & $\left(\bar{q}_{R i} \gamma_{\mu} q_{R j}\right)\left(\bar{\ell}_{R k} \gamma^{\mu} \ell_{R l}\right)$ & $O_{e d}, O_{e u}$ \\
$C_{V_{L R}}^{i j k l}$ & $\left(\bar{q}_{L i} \gamma_{\mu} q_{L j}\right)\left(\bar{\ell}_{R k} \gamma^{\mu} \ell_{R l}\right)$ & $O_{q e}$ \\
$C_{V_{R L}}^{i j k l}$ & $\left(\bar{q}_{R i} \gamma_{\mu} q_{R j}\right)\left(\bar{\ell}_{L k} \gamma^{\mu} \ell_{L l}\right)$ & $O_{l u}, O_{l d}$ \\
$C_{S_{R}}^{i j k l}$ & $\left(\bar{q}_{R i} q_{L j}\right)\left(\bar{\ell}_{L k} \ell_{R l}\right)+$ h.c. & $O_{l e d q}$ \\
$C_{S_{L}}^{i j k l}$ & $\left(\bar{q}_{L i} q_{R j}\right)\left(\bar{\ell}_{L k} \ell_{R l}\right)+$ h.c. & $O_{l e q u}^{(1)}$ \\
$C_{T}^{i j k l}$ & $\left(\bar{q}_{L i} \sigma_{\mu v} q_{R j}\right)\left(\bar{\ell}_{L k} \sigma^{\mu \nu} \ell_{R l}\right)+$ h.c. & $O_{l e q u}^{(3)}$ \\
\hline
\end{tabular}

Table 1: Operators $O_{\alpha}$ appearing in Eq. (1) and their corresponding operators in the SM EFT (third column). Flavor indices are denoted by $i, j, k, l$, and $q$ stands for either up or down-type quarks in the mass basis. Wilson coefficients are assumed to be real. See the Appendix of Ref. [11] for details.

where $\hat{s}$ denotes the partonic energy and $\hat{t} \in(-\hat{s}, 0)$. After integration over $\hat{t}$, we obtain

$$
[\hat{\sigma}(\hat{s})]_{i j k l}=\frac{\hat{s}}{144 \pi v^{4}} \sum_{\alpha \beta} C_{\alpha} C_{\beta}^{*} M_{\alpha \beta}
$$

where $\alpha, \beta \in\left\{V_{L L}, V_{R R}, V_{L R}, V_{R L}, S_{L}, S_{R}, T\right\}$ and $M_{\alpha \beta}$ is a matrix of numeric coefficients. In this equation, one should perform the following replacements

$$
C_{V_{X, Y}} \rightarrow C_{V_{X, Y}}^{i j k l}, \quad C_{S_{X}} \rightarrow \sqrt{\left|C_{S_{X}}^{i j k l}\right|^{2}+\left|C_{S_{X}}^{j i l k}\right|^{2}}, \quad C_{T} \rightarrow \sqrt{\left|C_{T}^{i j k l}\right|^{2}+\left|C_{T}^{j i l k}\right|^{2}},
$$

where $X, Y \in\{L, R\}$, and $M$ is a diagonal matrix $M_{\alpha \beta} \equiv \delta_{\alpha \beta} M_{\alpha}$, with $M=(1,1,1,1,3 / 4,3 / 4,4)$. The partonic cross-section should be convoluted with the relevant parton-parton luminosities [12], which in this work we define by the dimensionless functions

$$
\mathcal{L}_{q_{i} \bar{q}_{j}}(\tau)=\tau \int_{\tau}^{1} \frac{\mathrm{d} x}{x}\left[f_{q_{i}}\left(x, \mu_{F}\right) f_{\bar{q}_{j}}\left(\tau / x, \mu_{F}\right)+\left(q_{i} \leftrightarrow \bar{q}_{j}\right)\right],
$$

where $f_{q_{i}}$ denotes the quark $q_{i}$ parton distribution functions (PDF), $\mu_{F}$ is the factorization scale and $\sqrt{s}$ stands for the proton-proton center-of-mass energy, with $\tau=\hat{s} / s$. In our analysis, we consider the PDF4LHC15_nnlo_mc PDF set [13-16] and included the $1 \sigma$ PDF uncertainties derived by using the MC replica method [17]. The hadronic cross-section is then given by the expression

$$
\sigma\left(p p \rightarrow \ell_{k}^{-} \ell_{l}^{+}\right)=\sum_{i j} \int \frac{\mathrm{d} \tau}{\tau} \mathcal{L}_{q_{i} \bar{q}_{j}}(\tau)[\hat{\sigma}(\tau s)]_{i j k l},
$$

where the summation extends over all quark flavors, with the exception of the top quark which only contributes at one-loop to this process [18]. Note that if the partonic cross-section $\hat{\sigma}$ is a linear function in $\tau$, as it is our case, then the only dependence on $\tau$ of the integrand in Eq. (5) comes from the luminosity functions defined in Eq. (4). 

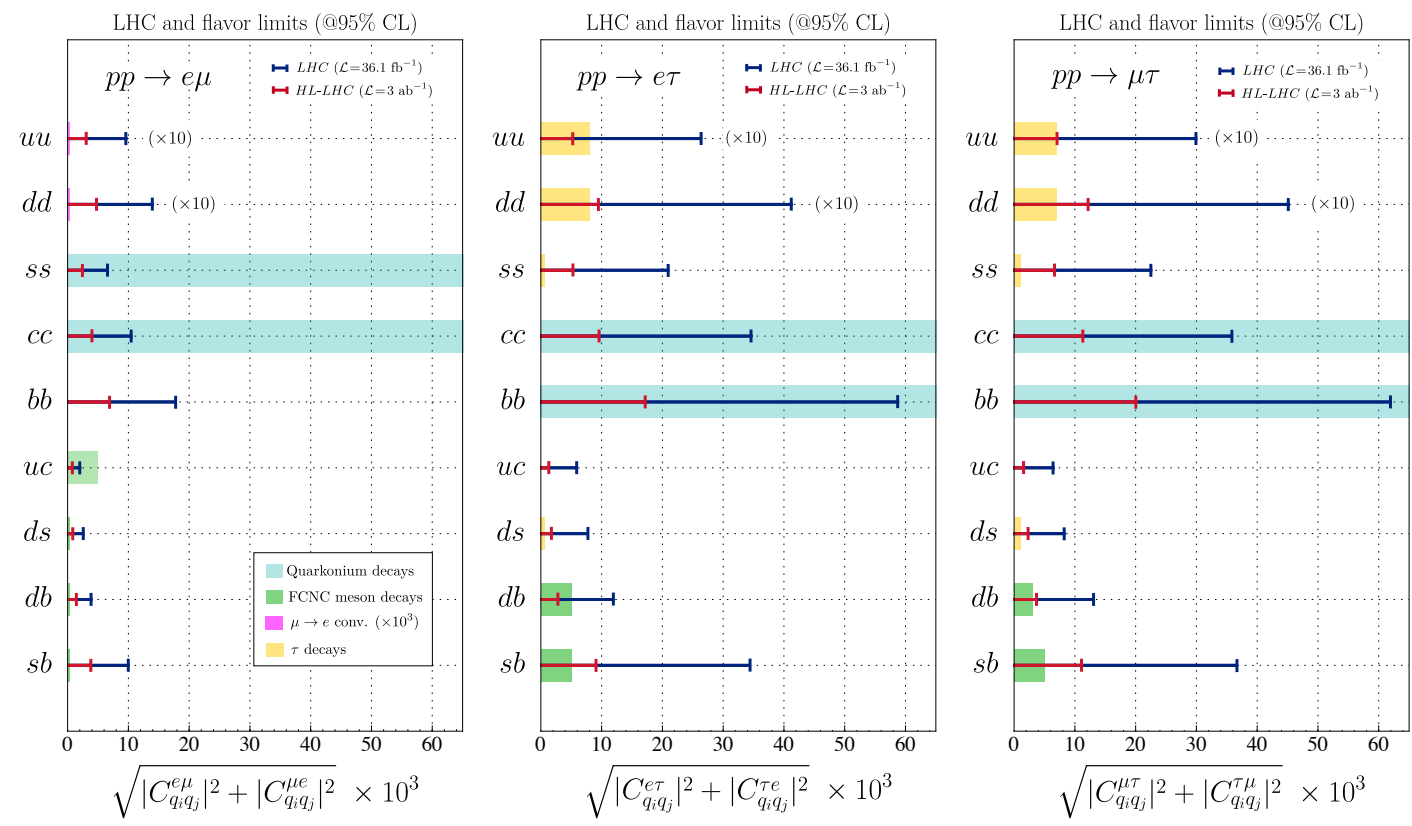

Figure 1: Limits derived from high-p $p_{T}$ LFV dilepton tails on the coefficients $\sqrt{\left|C_{q_{i}}^{\ell_{2} \ell_{j}}\right|^{2}+\left|C_{q_{i}}^{\ell_{1} \ell_{k}}\right|^{2}}$ by using

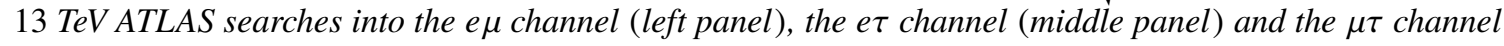
(right panel)s [19]. For comparison, we also show the limits obtained by the flavor physics observables, namely quarkonium decays (cyan), $\mu N \rightarrow e N$ (magenta), FCNC meson decays (green) and LFV $\tau$-decays (yellow). LHC and flavor results for $u u, d d \rightarrow e \mu, e \tau, \mu \tau$ have been rescaled by an additional factor of $\times 10$ for visibility. The limits from $\mu N \rightarrow e N$ have been rescaled by a factor of $\times 10^{3}$ to become visible.

\section{Recast of high- $p_{T}$ results}

We considered the latest ATLAS search for heavy vector resonances decaying into leptons with different flavors, $p p \rightarrow Z^{\prime} \rightarrow \ell_{k}^{ \pm} \ell_{l}^{\mp}$, performed at $\sqrt{s}=13 \mathrm{TeV}$ with $36.1 \mathrm{fb}^{-1}$ of data [19]. This search was recast for the final states $e \mu, e \tau$ and $\mu \tau$, following the procedure described in Ref. [11]. The bins of dilepton invariant mass $m_{\ell_{k} \ell_{l}}$ above $300 \mathrm{GeV}$ have been combined to extract 95\% confidence level (CL) upper limits on the effective coefficients defined in Eq. (1) for all possible quark-flavor combinations. Projections for the High-Luminosity LHC phase (HL-LHC) for a luminosity of $3 \mathrm{ab}^{-1}$ have been obtained by assuming that the data scales naively with the luminosity ratio and that all uncertainties scale with the square-root of the luminosity ratio. Our results are reported in Fig. 1 for the benchmark scenario defined by $C_{q_{i} q_{j}}^{\ell_{k} \ell_{l}} \equiv C_{V_{L L}}^{i j k l}$, where $i, j$ are flavor indices of down $(d, s, b)$ or up-type quarks $(u, c)$, and $k, l$ of charged leptons $(e, \mu, \tau)$, in the mass basis. Our definition is such that Hermiticity of Eq. (1) implies $C_{q_{i} q_{j}}^{\ell_{k} \ell_{l}}=\left(C_{q_{j} q_{i}}^{\ell_{l} \ell_{k}}\right)^{*}$.

\section{Discussion}

In Fig. 1, we compare the constraints we have derived from high- $p_{T}$ LHC data with the ones extracted from searches for low-energy LFV observables that depend, at tree-level, on the same effective couplings $C_{q_{i} q_{j}}^{\ell_{k} \ell_{l}}$. There are several types of low-energy observables that are relevant for our analysis [20]: (i) processes that also violate quark flavor, such as $K \rightarrow \pi \mu e, B_{s} \rightarrow \ell_{k} \ell_{l}$ and 
$B \rightarrow K^{(*)} \ell_{k} \ell_{l}$; (ii) quarkonia decays, such as $J / \psi \rightarrow \ell_{k} \ell_{l}$ and $\Upsilon \rightarrow \ell_{k} \ell_{l}$; (iii) $\tau$-decays, such as $\tau \rightarrow \mu \phi$ and $\tau \rightarrow \mu \pi$; and (iv) $\mu \rightarrow e$ conversion in nuclei. Several conclusions can be derived from Fig. 1:

- We find that high- $p_{T}$ constraints are significantly better than low-energy observables for quark-flavor conserving coefficients such as $C_{c c}^{l k}$ and $C_{b b}^{l k}$, for all lepton flavors. The same conclusion also holds for $C_{s s}^{e \mu}$. This is the case because the low-energy observables that can probe these coefficients are quarkonia decays $(\phi, J / \psi$ and $\Upsilon$ ), which are highly suppressed by the large quarkonia decay width since these particles are flavor singlets.

- Our high- $p_{T}$ results are the only available constraints for the transitions $c \rightarrow u e \tau$ and $c \rightarrow u \mu \tau$, since there are no kinematically allowed flavor processes that can probe these transitions in exclusive meson decays.

- For quark-flavor violating processes, such as kaon and $B_{(s)}$-meson LFV decays, flavor limits are in general much more constraining than our high- $p_{T}$ limits. The only exceptions are the $b \rightarrow \operatorname{de\tau }$ and $b \rightarrow d \mu \tau$ transitions, for which the HL-LHC projections are comparable to the current sensitivity on the decays $B \rightarrow \pi e \mu$ and $B \rightarrow \pi \tau \mu$, as well as on the related leptonic modes [20].

In conclusion, there is a striking complementarity between the LFV limits that can be extracted from low-energy searches and from the study of high- $p_{T}$ tails, as explored in Ref. [11]. Same conclusions have also been obtained for flavor-conserving transitions, which can be constrained by the high- $p_{T}$ tails of $p p \rightarrow \ell \ell$ [8] and $p p \rightarrow \ell \bar{v}$ [9]. This complementarity was highlighted in this proceedings for left-handed operators, but it also holds to a smaller extent for scalar and tensor operators. These results illustrate that the combination of indirect low and high-energy searches is of fundamental importance in order to efficiently probe New Physics scenarios in the flavor sector.

\section{Acknowledgments}

This project has received funding from the European Research Council (ERC) under the European Union's Horizon 2020 research and innovation programme under grant agreement 833280 (FLAY), and by the Swiss National Science Foundation (SNF) under contract 200021-175940.

\section{References}

[1] S. Mihara, J. P. Miller, P. Paradisi and G. Piredda, Ann. Rev. Nucl. Part. Sci. 63 (2013), 531-552.

[2] L. Calibbi and G. Signorelli, Riv. Nuovo Cim. 41 (2018) no.2, 71-174 [arXiv:1709.00294 [hep-ph]].

[3] D. Bečirević, N. Košnik, O. Sumensari and R. Zukanovich Funchal, JHEP 11 (2016), 035 [arXiv:1608.07583]; D. Bečirević, O. Sumensari and R. Zukanovich Funchal, Eur. Phys. J. C 76 (2016) no.3, 134 [arXiv:1602.00881]. 
[4] E. Cortina Gil et al. [NA62], JINST 12 (2017) no.05, P05025 [arXiv:1703.08501 [physics.insdet]].

[5] M. Ablikim et al. [BESIII], Chin. Phys. C 44 (2020) no.4, 040001 [arXiv:1912.05983 [hepex]].

[6] R. Aaij et al. [LHCb], [arXiv:1808.08865 [hep-ex]].

[7] E. Kou et al. [Belle-II], PTEP 2019 (2019) no.12, 123C01 [erratum: PTEP 2020 (2020) no.2, 029201] [arXiv:1808.10567 [hep-ex]].

[8] D. A. Faroughy, A. Greljo and J. F. Kamenik, Phys. Lett. B 764 (2017), 126-134 [arXiv:1609.07138]; A. Greljo and D. Marzocca, Eur. Phys. J. C 77 (2017) no.8, 548 [arXiv:1704.09015].

[9] J. Fuentes-Martin, A. Greljo, J. Martin Camalich and J. D. Ruiz-Alvarez, JHEP 11 (2020), 080 [arXiv:2003.12421]; D. Marzocca, U. Min and M. Son, [arXiv:2008.07541]; S. Iguro, M. Takeuchi and R. Watanabe, [arXiv:2011.02486].

[10] D. Buttazzo, A. Greljo, G. Isidori and D. Marzocca, JHEP 11 (2017), 044 [arXiv: 1706.07808]; A. Angelescu, D. Bečirević, D. A. Faroughy and O. Sumensari, JHEP 10 (2018), 183 [arXiv:1808.08179].

[11] A. Angelescu, D. A. Faroughy and O. Sumensari, Eur. Phys. J. C 80 (2020) no.7, 641 [arXiv:2002.05684].

[12] J. M. Campbell, J. W. Huston and W. J. Stirling, Rept. Prog. Phys. 70 (2007), 89 [arXiv:hep$\mathrm{ph} / 0611148]$.

[13] J. Butterworth, S. Carrazza, A. Cooper-Sarkar, A. De Roeck, J. Feltesse, S. Forte, J. Gao, S. Glazov, J. Huston and Z. Kassabov, et al. J. Phys. G 43 (2016), 023001 [arXiv:1510.03865].

[14] R. D. Ball et al. [NNPDF], JHEP 04 (2015), 040 [arXiv:1410.8849].

[15] L. A. Harland-Lang, A. D. Martin, P. Motylinski and R. S. Thorne, Eur. Phys. J. C 75 (2015) no.5, 204 [arXiv:1412.3989].

[16] S. Dulat, T. J. Hou, J. Gao, M. Guzzi, J. Huston, P. Nadolsky, J. Pumplin, C. Schmidt, D. Stump and C. P. Yuan, Phys. Rev. D 93 (2016) no.3, 033006 [arXiv:1506.07443].

[17] S. Carrazza, J. I. Latorre, J. Rojo and G. Watt, Eur. Phys. J. C 75 (2015), 474 [arXiv:1504.06469].

[18] B. Bhattacharya, R. Morgan, J. Osborne and A. A. Petrov, Phys. Lett. B 785 (2018), 165170 [arXiv:1802.06082]; Y. Cai, M. A. Schmidt and G. Valencia, JHEP 05 (2018), 143 [arXiv:1802.09822].

[19] M. Aaboud et al. [ATLAS], Phys. Rev. D 98 (2018) no.9, 092008 [arXiv:1807.06573 [hep-ex]].

[20] P. A. Zyla et al. [Particle Data Group], PTEP 2020 (2020) no.8, 083 C01. 